\title{
Challenges and Perspectives of Omes \& Omics in Assisted Reproductive Technologies: From Screening to Diagnostic
}

\author{
Moncef Benkhalifa*1, Naima Belhadri ${ }^{1}$, Stephanie Belloc ${ }^{2}$, Rosalie Cabry ${ }^{1}$, Hikmat Chahine ${ }^{3}$, Henri \\ Copin ${ }^{1}$, Pierre Miron ${ }^{4}$ and Timur Gurgan ${ }^{5}$ \\ ${ }^{1}$ ART \& Reproductive Genetics and PERITOX Laboratory, Picardie University Jules Verne. Amiens. France
}

${ }^{2}$ Laboratoire Lavergne ACCOLAB, France

${ }^{3}$ Laboratoire FORTEBIO/UNILABS, France

${ }^{4}$ Centre d'aide médicale à la procréation FERTILYS. Laval, Canada

${ }^{5}$ ART \& genetics. Gurgan Clinic Ankara and Bahcesehir University, Turkey

Received: June 6, 2018; Published: June 14, 2018

*Corresponding author: Moncef Benkhalifa, ART \& Reproductive Genetics and PERITOX Laboratory. Regional University Hospital \& School of Medicine. CURS. Picardie University Jules Verne. Amiens. France

\section{Mini review}

In cellular physiology and functional biology, the suffix -ome refers to "all constituents considered together and as a mix". During the last decade the omics development have significantly changed the face of fundamental and clinical biology for diagnostics and clinical treatment. By building bridges to cell biology, Omics technologies are no longer limited any more to research laboratories but started to be applied in clinical biology for prognostic and diagnostic [1]. For example, fast moving advances in next generation sequencing technologies makes human sequence datasets widely available and this represents a fundamental shift from basic research to clinical application. Genomics has already moved to the clinic and combining medical records with genome sequences offers tantalizing possibilities for the future of medicine [2]. IVF omes and omics are becoming promising technologies that personalize patient management and improve success rates $[3,4]$.

In reproductive pathology, the contribution of genome and epigenome defects such as chromosomes abnormalities, genes and/or metabolic disorders and functional biology process misfit are very well documented as a main factor of assisted reproductive technology cycles failures. General survey showed in somatic karyotype a higher incidence of chromosomal aberrations in infertile and sub fertile couples than neonatal population. For example, cytogenetic studies of infertile male revealed between 2 to 15 of abnormalities. In azoospermia cases, nearly $15 \%$ are carrier of unbalanced karyotypes. In oligospermia cases, chromosomes disorders were observed in only 2 to $5 \%$. More than numerical abnormalities balanced and unbalanced abnormalities involving autosomes-gonosomes chromosomes are also reported [5].

In IVF programmes, risk assessment and safety management need to be analyzed with care to minimize failure and adverse effects [6]. In Assisted Reproductive Technology practice, gametes maturation and competency (genic and epigenetic levels) are mandatory for fertilization, early embryo cleavage and development success. Ovarian stimulation with gonadotropins can improve the quantity of collected gametes but without full guarantee of quality. To better assess, select and predict gametes and embryo quality, cytological, cytogenetics, molecular techniques (karyotype, FISH, PCR and sequencing) or imaging methods have been routinely applied over the last few years. For example, in severe male infertility and ICSI failure, the investigation of sperm genome decays (chromosome abnormalities, DNA fragmentation, chromatin denaturation and methylome changes $[4,7,8]$ is currently performed to explain the potential contribution of sperm on fertilization failure and embryo cleavage blockage.

In parallel, oocyte imaging, genome analysis and mitochondria functionality assessment can also be helpful to predict metaphase II oocyte maturation and chromosome disjunction from patient with polycystic ovary syndrome or to assess effect of ovarian stimulation protocols [9-11]. Using different techniques, the literature showed clearly that abnormal sperm and/or oocyte genome or epigenome can be major factors of oocyte maturation and activation underachievement, early embryo blockage, implantation failure, miscarriage and abnormal birth [12-14]. For the last 25 years, preimplantation genetic diagnosis was one of the major application of genomics in ART to diagnose chromosome and genome integrity abnormalities or specific gene disorders to eliminate the 
worst embryo prior transfer and improve clinical pregnancy and taking home baby. For many years, techniques such as multicolor FISH, PCR, single point mutation or fragment sizing were used to investigate polar bodies, blastomeres or trophectoderm cells.

Recently, Genomic or expression microarray technology, Comprehensive chromosome screening and new generation sequencing are becoming common tools for genome and gene investigation in research and development and in clinical diagnostic to predict and select the best gametes and embryo. For genome profiling and high resolution molecular karyotyping, array comparative genome hybridization and sequencing methods appears to be far better and have much higher sensitivity and specificity for subtle genomic changes [15-20]. In reproductive medicine and IVF, Nano genomics and omes technologies (genomic, expression or proteins arrays) will be an important clinical assay for genomics, transcriptomics and proteomics testing. As noninvasive tests, cumulus and endometrial cells transcriptome profiling combined with metabolic monitoring of culture media can give valuable information on embryo implantation competency and IVF success rate improvement $[10,12]$.

Compared to genome analysis, transcriptome profiling can be different between different cell types, developmental stage and depends on stimulation protocol or gonadotropins types. In oligoazooteratospermia, the transcriptome investigation of sperm cell can explain certain decays related to the maintenance of transcripts integrity which is mandatory for oocyte activation after fertilization [21]. It was also reported that the transcriptome of oocytes can be different from one ovarian stimulation protocols to another and the proteomic profile of immature oocytes is totally different than oocyte maturated in vitro and this difference can be mustered during the maturation process. Also, proteomics showed that the female oviduct has a specific system to recognize the gamete and allow modification of the secretome environment in the presence of male and female gametes $[22,23]$.

Proteome and metabolome analysis of early embryo can be more informative than the actual techniques currently used in the laboratory to assess embryo quality based on the morphology and cleavage kinetic. It was clearly demonstrated that there is a synchronism between the morphology and the biological implantation competency of the embryo [24]. The improvement of those tools in ART will give us more information closer the functional biology of the gametes and the embryo. The comparison of the transcriptome and/or proteome signature of the embryo and the endometrium, prior to the transfer, can be useful to select the most competent embryo for implantation and assess the best synchronized endometrium for transfer. Thanks to this approach, sequential IVF become a reality with improvement of tissue, gamete and embryo cryopreservation (slow freezing and vitrification). By combining all different technologies with sequential management, specialists are improving day by day clinical outcomes and they are managing more difficult cases.

On a daily basis, there is a still technical and conceptual limitations of omes and omics technology to be applied routinely in
ART. For genomic micro arrays use, it is important to consider the continued dynamic genome changes and interpret with care copy number variants and variants of unknown significance (CNV and VOUS). Quantification of expressions levels in single cell or in group of cells can reflect the transcriptional state and therefore differential expression patterns of the proteome. We know that proteins are modified after translation, from simple phosphorylation or acetylation to complex glycoprotein structures, and that this cascade of events complicates investigation and analysis of the embryo metabolome. The complicate functional cascade from gene to metabolome and cellular development from gamete to blastocyst are opening a new area of investigation mainly epigennomics and methylation profiling of gametes and products of conception [25].

Concerning the window of implantation, interact omics between the blastocyst and the endometrium will soon be a fastgrowing field of omics by linking the interaction between molecules produced by embryo and endometrial cells, as will be regulomics, based on the study of regulation of the molecules involves in the implantation. A wide range of molecular methods are more and more required for advanced screening and diagnostic and for better understanding of reproductive pathology causes. For example, application of autosomal gene targeting technologies showed clearly that several hundred of candidate genes are involved in gametogenesis pathophysiology. Molecular technology improvement such as digital PCR, high throughput and new generation sequencing on parallel to technology transfer from research benches to diagnostics will open a new era in infertility investigation and management. Circulating or isolated single somatic or germinal cell for phenotype or genotype analysis is a promising approach for fertility investigation.

Challenges will be resolved through a democratic access to first class molecular technologies and a reduction in daily practice costs (Economics); Only then, a real window of application of Omes and Omics for investigation, diagnostics and therapeutics in Assisted Reproductive Technology (ART) will be opened at different levels of testings for screening and diagnostics [5]. Perspectives application of omes and omics in ART will be the noninvasive tools [26-29] to investigate gametes fertility potential, embryos development capacity and endometrium receptivity in order to improve clinical outcome and taking home baby.

\section{References}

1. Cox B (2009) Building bridges from omics to cell biology. Genome Biol 10(3): 305.

2. Webb S (2012) Genomics moves to the clinic. Biotechniques 52(2): 7376.

3. Hamamah S (2011) Omics as a tool for ART. Gyn/Obs \& fertyl 39:1-2.

4. Brown R, Harper C (2012) The clinical benefit and safety of current and future assisted reproductive technology. MBM Online 25: 108-117.

5. Benkhalifa M, Montjean D, Belloc S, Dalleac A, Ducasse M, et al. (2014) Emerging molecular methods for male infertility investigation. Expert Rev Mol Diagn 14(1): 37-45.

6. Meldrum D, Dezieglzer D (2013) Introduction: Risk and safety management in infertility and assisted reproductive technology. Fertil Steril 100(6): 1497-1498. 
7. Kovak R, Pastuszak W, Lamb D (2013) The use of genomics, proteomics, and metabolomics in identifying biomarkers of male infertility. Fertil Steril 99(4): 998-1007.

8. Montjean D, Ravel C, Benkhalifa M, Cohen Bacrie P, Berthaut I, Bashamboo A, et al. (2013) Methylation changes in mature sperm deoxyribonucleic acid from oligozoospermic men: assessment of genetic variants and assisted reproductive technology outcome. Fertil Steril 100(5): 12411247.

9. Huang y, Rosenwaks Z (2012) In vitro fertilization treatments and factors affecting success. Best Pract Rese Clin Obstet Gynaecol 26(6): 777-788.

10. Assou S, Haouzi D, Dechaud H, Gala A, Ferrières A, et al. (2013) Comparative gene expression profiling in human cumulus cells according to ovarian gonadotropin treatments. Biomed Res Int 12: 35-45.

11. Labrecque R, Sirard M (2014) The study of mammalian oocyte competency by transcriptome analysis: progress and challenges. Mol Hum Reprod 20(2): 103-116.

12. Oundaogo Z, Haouzi D, Assou S, Dechaud H, Kadoch J, et al. (2011) Human cumulus cells molecular signature in relation to oocyte nuclear maturity stage. PloS One 6(11): 271-179.

13. Nel Themmat L, Nagy P (2011) A review of the promises and pitfalls of oocyte and embryo metabolomics. Placenta 32(S3): 257-263.

14. Collins C (2013) Primplantation genetics diagnosis: technical advances and expanding applications. Curr Opin Obstet Gynecol 25(3): 201-206.

15. Scren P, Ogilvie C, Khalaf Y (2012) Embryo selection in IVF: Is polar body array comparative genomic hybridization accurate enough; Hum Repro 27(4): 951-953.

16. Treff N (2012) Genome wide analysis of human preimplantation aneuploidy. Semin Reprod Med 30(4): 283-288.

17. Scott R, Ferry K, Su J, Tao X, Scott K, et al. (2012) Comprehensive chromosome screening is highly predictive of the reproductive potential of human embryos: a prospective, blinded nonselection study. Fertil steril 97(4): 870-875.

18. Martin j, Cervero A, Mir P, Martinez Conejero J, Pellicer A, et al. (2013) The impact of next generation sequencing technology on preimplantation genetic diagnosis and screening. Fertile Steril 99(4): 1054-1061.
19. Tan Y, Tan K, Zhang S, Gong F, Cheng D, et al. (2013) Single nucleotide polymorphisme micro array based preimplantation genetic diagnosis is likely to improve the clinical outcome for translocation carriers. Hum repro 28(9): 2581-2592.

20. Rubio C, Rodrigo L, Mir P, Mateu E, Peinado V, et al. (2013) Use of array comparative genomic hybridization (array-CGH) for embryo assessment: clinical results. Fertil Steril 99(4): 1044-1048.

21. Montjean D, De La Grange P, Gentien D, Rapinat A, Belloc S, et al. (2012) Sperm transcriptome profiling in oligozoospermia. J Assist Reprod Genet 29(1): 3-10.

22. Bianchi L, Gagliardi A, Campanella G, Landi C, Capaldo A, et al. (2013) A methodological and functional proteomic approach of human follicular fluid en route for oocyte quality evaluation. J Proteomics 90: 61-76.

23. Nyalwidhe j, Burch T, Bocca S, Cazares L, Green Mitchel S, et al. (2013) The search for biomarkers of uman embryos developmental potential in IVF: a comprehensive proteomic approach. Mol Hum Reprod 19(4): 250-253.

24. Courant F, Antignac J, Monteau F, Le Bizec B (2013) Metabolomics as a potential new approach for investigating human reproductive disorders. J Proteome Res 12(6) : 2914-2920.

25. Lliadou A, Janson P, Cnattingius S (2011) Epigenetics and assisted reproductive technology. J Intern Med 270(5): 414-420.

26. Cohen J, Grudzinskas, Johnson M (2013) Embryonic DNA sampling without biopsy: the beginning of noninvasive PGD. RBM Online 26(6): 520-521.

27. Brison D, Hollywood K, Arnesen R, Goodacre R (2007) Predicting human embryo viability: the road to noninvasive analysis of the secretome using matbolic footprinting. RBM Online 15(3): 296-302.

28. SenGupta S, Delhanty J (2012) Preimplantation genetic diagnosis: recent triumphs and remaining challenges. Expert Rev Mol Diagn 12(6): 585592.

29. Cheong Y, Boomsma C, Heijnen C, Macklon N (2013) Uterine secretomics: a windo on the maternal embryo interface. Fertil Steril 15(4): 10931099.

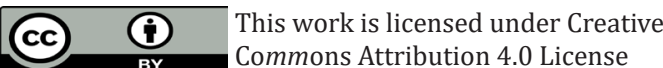

Submission Link: https://biomedres.us/submit-manuscript.php

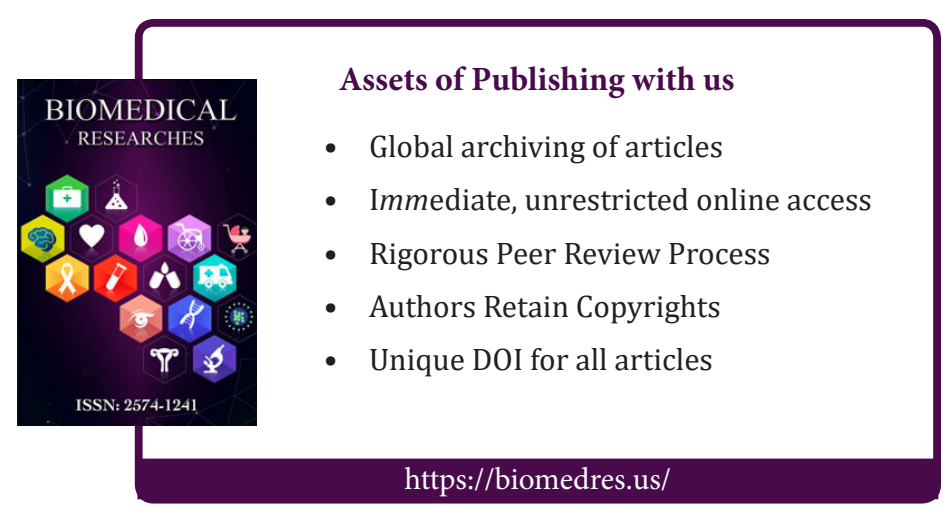

